\title{
EVALUATION OF BIOLOGICAL ACTIVITIES OF EVOLVULUS NUMMULARIUS L. THROUGH INSECTICIDAL, INSECT REPELLENCY AND BRINE SHRIMP LETHALITY TESTS
}

\author{
Md. Moniruzzaman, Nayeema Parvin, Sabina Sultana, Mohammad Abdullah, Ataur Rahman \\ Khan and Nurul Islam*
}

\author{
Department of Zoology, University of Rajshahi, Rajshahi-6205, Bangladesh \\ "Corresponding Author: e-mail: n_islamm@yahoo.com
}

\begin{abstract}
Assessment of Evolvulus nummularius L. through whole plant extracts (collected in petroleum ether [Pet.E.], chloroform [CHCl ${ }_{3}$ ] and methanol $[\mathrm{MeOH}]$ ) were made against Tribolium castaneum (Hbst.) for insecticidal and insect repellency and against Artemia salina L. for brine shrimp lethality, and the results were promising. The $\mathrm{LC}_{50}$ values against $T$. castaneum adults for Pet.E. extract were 1.280, 1.220, 1.096 and $1.030 \mu \mathrm{g} \mathrm{cm}^{-2}$; for $\mathrm{CHCl}_{3}$ extract 3.249, 2.990, 2.526 and $2.204 \mu \mathrm{g} \mathrm{cm}^{-2}$; and for MeOH extract 2.925, 1.899, 1.875 and $1.789 \mu \mathrm{g} \mathrm{cm} \mathrm{cm}^{-2}$, respectively for $12,24,36$ and $48 \mathrm{~h}$ of exposure. The intensity of activity could be arranged in a descending order: Pet.E. extract $>\mathrm{MeOH}$ extract $>\mathrm{CHCl}_{3}$ extract. For repellency, $\mathrm{CHCl}_{3}$ and $\mathrm{MeOH}$ extracts offered moderate activity $(\mathrm{P}<0.01)$, but the Pet.E. extract did not show efficacy against the beetles. The same extracts responded positively in brine shrimp lethality assay where the $\mathrm{LC}_{50}$ values for Pet.E. extract were $141.553,98.941$, 24.684 and $8.560 \mathrm{ppm}$; for $\mathrm{CHCl}_{3}$ extracts 699.048, 366.663, 326.0039 and 40.114ppm; and for $\mathrm{MeOH}$ extracts 2336.982, 1258.605, 355.962 and $137.0589 \mathrm{ppm}$ respectively for $12,18,24$ and $30 \mathrm{~h}$ of exposure. However, the intensity of activity could be arranged in a descending order: Pet.E. extract $>\mathrm{CHCl}_{3}$ extract $>\mathrm{MeOH}$ extract.
\end{abstract}

Keywords: Evolvulus nummularius; dose-mortality, repellency, brine shrimp lethality, Tribolium castaneum, Artemia salina.

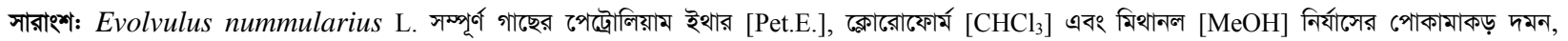
পোকামাকড় বিতাড়ন কার্যকারিতা যাচাইয়ের জন্য গমের কেড়িপোকা Tribolium castaneum (Herbst) এর পূর্ণাঙ্গ দশার উপর ও মারণ কার্যকর্ाারতা যাচাইট়ের জন্য Artemia salina L. এর উপর গবেষণাগার ব্যবস্থায় পরীক্ষা চালান হয়, এবং আশাপ্রদ ফলাফল পাওয়া যায়। এক্ষেত্রে Pet.E. নির্যাসটির বিপরীতে $\mathrm{LC}_{50}$ ছিল যথাক্রমে ১.২৮০, ১.২২০, ১.০৯৬ এবং ১.০৩০ মাইক্রোগ্রাম প্রতি বর্গসেন্টিমিটারে আর $\mathrm{CHCl}_{3}$ নির্यাসটির বিপরীতে $\mathrm{LC}_{50}$ ছিল যথাক্রমে ৩.২৪৯, ২.৯৯০, ২.৫২৬ এবং ২.২০৪ মাইক্রোগ্রাম প্রতি বর্গসেন্টিমিটারে; অবশ্য $\mathrm{MeOH}$ নির্যাসটির বিপরীতে ছিল যথাক্রমে ২.৯২৫, ১.৮-৯৯, ১.৮-৭৫ ও ১.৭৮-৯ মাইক্রোগ্রাম প্রতি বর্গকেন্টিমিটারে এবং সবক্ষেত্রে পাঠ নেয়া হয়েছে ১২, ২৪, ৩৬ ও ৪b ঘন্টায়। নিম্নক্রমানুসারে সাজালে কার্যকারিতা ফলাফল দাঁড়ায় Pet.E. নির্যাস> $\mathrm{MeOH}$ निर्याস> $\mathrm{CHCl}_{3}$ निর্যাস। পূর্ণাঙ $T$. castaneum এর উপর $\mathrm{CHCl}_{3}$ ও $\mathrm{MeOH}$ निर्याস বিতাড়ন কার্যকারিতা প্রদর্শন করেছে ১\% তাৎপর্যতায় $(\mathrm{P}<0 . ০ ১)$, আর Pet.E. নির্यাস কোনরূপ কার্যকারিতা দেখায়নি। একই নির্यাসগুলি brine shrimp lethality পরীক্ষণে বির্যক্রিয়া দেখিয়েছে। এক্ষেত্রে Pet.E. নির্यাসটির বিপরীতে LC L $_{50}$ ছিল যথাক্রনে ১8১.৫৫৩, ৯৮.৯8১, ২8.৬b-8 ও ৮.৫৬০ পিপিএম; $\mathrm{CHCl}_{3}$ निর্যাসটির বিপরীতে যথাক্রনে ৬৯৯.০৪b, ৩৬৬.৬৬৩, ৩২৬.০০৩৯ ও 8০.১১৪ পিপিএম; এবং $\mathrm{MeOH}$ নির্যাসটির বিপরীতে যথাক্রনে ২৩৩৬.৯৮-২, ১২৫৮.৬০৫, ৩৫৫.৯৬২ ও ১৩৭.০৫৮-৯ পিপিএম এবং সবগুলির ক্ষেত্রে পাঠ নেয়া হয়েছে ১২, ১৮-, ২৪ ও ৩০ ঘন্টায়। নিম্নক্রমানুসারে সাজালে কার্যকারিতা ফলাফল দাঁড়ায় Pet.E. निर्याস> $\mathrm{CHCl}_{3}$ निर्याস> $\mathrm{MeOH}$ निर्याস।

\section{Introduction}

Evolvulus nummularius L. (=Convolvulus nummularius L.; Roundleaf Bindweed; ভুঁই আকড়া) (F. Convolvulaceae). It was taken into consideration for screening of its biological activities. This plant is found in Africa, Bangladesh, India, Malaysia and Myanmar. In Bangladesh it is found in all the districts. Medicinal plants are the most important source of lifesaving drugs for the majority of the world population. These plants are moving from fringe to mainstream use, as a greater number of people endeavour to opt for herbal formulations over the allopathic compounds, since these are devoid of side effects and cost effective (Dubey et al., 2004). Popular demand and scientific interest in complementary or alternative medicine, particularly medicinal botanicals, has increased considerably since mid-90's (Borchers et al., 1997). Screening and indexing of many plants, which have been used in traditional Indian and Chinese medicines since time immemorial, resulted in novel therapeutics, useful for the treatment of various ailments of man, such as rheumatism, kapha, pitha, blood pressure and cancer (Anon, 1948; Anon, 1959). In this investigation E. nummularius L. had been selected against the red flour beetle, Tribolium castaneum (Hbst.) for its toxic and repellent activity, and Artemia salina L. nauplii for lethality test for the detection of its biological activities.

\section{Materials and Methods}

Collection and preparation of test materials: Fresh plants were collected from the Rajshahi University Campus, Bangladesh, identified and a voucher specimen (No. 60, 10-05-1983) was kept in the herbarium of the Department of Botany, University of Rajshahi. After collection fresh plants were chopped into small pieces to dry under shade in a well-ventilated room. Dried materials were then powdered using a hand grinder, weighed and placed in a conical flask $(500 \mathrm{ml})$ to add petroleum ether (Pet.E.) $(100 \mathrm{gm} \times 300 \mathrm{ml} \times$ 2 times) for $48 \mathrm{~h}$. Filtration was done by Whatman filter paper at $24 \mathrm{~h}$ interval in the same flask followed by evaporation until the extract ware left. The same material was then placed in another conical flask to add 
chloroform $\left(\mathrm{CHCl}_{3}\right)$ and the same done for methanol $(\mathrm{MeOH})$ extraction. The extracts were then transferred to glass vials and preserved in a refrigerator at $4{ }^{\circ} \mathrm{C}$ with proper labeling.

Collection and culture of test insects: $T$. castaneum beetles were reared in glass beakers $(500 \mathrm{ml})$ in a standard mixture of whole-wheat flour with powdered dry yeast (19:1) in an incubator at $30^{\circ} \mathrm{C} \pm 0.5^{\circ} \mathrm{C}$ without light and humidity control, for a continuous supply of adults during experimentation.

Collection and culture of brine shrimp nauplii: Eggs (cyst) of A. salina were purchased from aquarium shops of Kalabagan, Dhaka and kept in aerated seawater (with $3.8 \%$ sodium chloride or $38 \mathrm{gm}$ salt $/ 1000 \mathrm{ml}$ of pond water) at room $\left(25-30^{\circ} \mathrm{C}\right)$ temperature. It normally takes $30-48 \mathrm{~h}$ to give nauplii under these conditions. Freshlyhatched nauplii were used in this experiment.

Dose-mortality test against $T$. castaneum: For dosemortality responses by surface film method, different doses of the extracts of Pet.E., $\mathrm{CHCl}_{3}$ and $\mathrm{MeOH}$ of $E$. nummularius (whole plant) were selected through ad hoc experiments by diluting $50 \mathrm{mg}$ of each of the extracts separately in $1 \mathrm{ml}$ of solvent to apply in $50 \mathrm{~mm}$ Petri dishes and by increasing or decreasing the amount of extracts in repeated manner until a suitable mortality range was obtained. For the Pet.E. extract a general concentration was selected to produce other successive doses by serial dilution to give $1.529,1.274,1.019$, $0.764,0.510$ and $0.255 \mu \mathrm{g} \mathrm{cm}^{-2}$. For the $\mathrm{CHCl}_{3}$ extract, a general concentration was selected to give 4.076, 3.567, $3.057,2.548$ and $2.038 \mu \mathrm{g} \mathrm{cm}^{-2}$. For the $\mathrm{MeOH}$ extract, a general concentration to give $3.567,3.057,2.548$, 2.038 and $1.529 \mu \mathrm{g} \mathrm{cm}^{-2}$. Each of the doses were diluted in $1 \mathrm{ml}$ of the respective solvent poured into Petri dishes and allowed to dry. Ten adult beetles were released in each Petri dish, and the experiment of all the doses for each of the extracts were set in 3 replicates. The mortality of the beetles was assessed at 12, 24, 36 and $48 \mathrm{~h}$ of exposures.

Statistical analysis of the dose-mortality data: The mortality of the beetles was recorded, while an instant observation was made just after 30 min. of application for the detection of acute toxicity, if any. The mortality (\%) was corrected by Abbott's formula (1925). The statistical analyses were done according to Finney (1947) and Busvine (1971) to calculate the $\mathrm{LC}_{50}$ values by using a 'software' developed in the Department of Agricultural and Environmental Science, University of Newcastle upon Tyne, U.K.
Repellent activity against $T$. castaneum adults: The methodology for repellency test used in this experiment was adopted from the method (No. 3) of McDonald et al. (1970) with some modifications by Talukder and Howse (1993, 1994). Half filter paper discs (Whatman No. 40 , diameter $9 \mathrm{~cm}$ ) were treated with the selected doses of $10,5,2.5,1.25$ and $0.625 \mathrm{mg} \mathrm{cm}^{-2}$ of Pet.E. extract and were then attached lengthwise, edge-to-edge to a control half-disc with adhesive tape and placed in the Petri dishes. The orientation was changed in the two remaining replicates to avoid the effects of any external directional stimulus affecting the distribution of the test insects. Ten adult insects were released in the middle of each of the filter paper circles. The same was then done for the $\mathrm{CHCl}_{3}$ and $\mathrm{MeOH}$ extracts.

Analysis of repellency data: Each concentration was tested for five times. Insects that settled on each of the non-treated half of the filter paper discs were counted after $1 \mathrm{~h}$ and then observed repeatedly at hourly intervals for 5 hours. The average of the counts was converted to percent repulsion $(P R)$ using the formula of Talukder and Howse (1995): $P R=(\mathrm{Nc}-5) \times 20$, where, $\mathrm{Nc}$ is the percentage of insects on the untreated half of the disc.

Lethality against brine shrimp nauplii: For Pet.E. extract a $5 \mathrm{mg}$ sample was initially dissolved in $250 \mu \mathrm{l}$ of dimethyl sulfoxide (DMSO) to make it hydrophilic before adding $5 \mathrm{ml}$ of water and diluted up to $10 \mathrm{ml}$ with brine water. A similar dose was set to obtain a series of concentrations, viz. 475.9, 237.5, 118.7, 59.3 and $29.6 \mathrm{ppm}$ as other doses. In this way a general concentration of $\mathrm{CHCl}_{3}$ extract was selected to give 499.5, 249.7, 124.8, 62.5 and 31.2ppm and for $\mathrm{MeOH}$ extract a general concentration was selected to give 572.5, 286.6, 143.5, 71.5 and 35.7ppm. Each concentration was tested in triplicate. Ten freshly hatched nauplii were added to each of the test tubes with different concentrations mentioned above. The lethality of the nauplii to the extracts was observed after $12,18,24$ and $30 \mathrm{~h}$ of exposure by counting the number died in each of the test tubes. The data was then subjected to probit analysis to get $\mathrm{LC}_{50}$ values.

\section{Results and Discussion}

Dose-mortality against $T$. castaneum adults: The results of the dose-mortality assay and replellency of Pet. E., $\mathrm{CHCl}_{3}$ and $\mathrm{MeOH}$ extracts against $T$. castaneum adults are represented in Table 1 . The $\mathrm{LC}_{50}$ values for the Pet.E. extract ranged between 1.280 to $1.030 \mu \mathrm{g} \mathrm{cm}^{-2}$; for the $\mathrm{CHCl}_{3}$ extract it ranged between 3.249 to $2.204 \mu \mathrm{g} \mathrm{cm}^{-2}$ and for the $\mathrm{MeOH}$ extract it was 2.925 to $1.789 \mu \mathrm{g} \mathrm{cm}^{-2}$. 
Table $1 \mathrm{LC}_{50}$ values of Pet.E., $\mathrm{CHCl}_{3}$ and $\mathrm{MeOH}$ extracts of $E$. nummularius whole plant against T. castaneum adults.

\begin{tabular}{|c|c|c|c|c|c|c|c|}
\hline \multirow{2}{*}{$\begin{array}{l}\text { Type of } \\
\text { extract }\end{array}$} & \multirow{2}{*}{$\begin{array}{c}\text { Time } \\
\text { exposed }\end{array}$} & \multirow{2}{*}{$\begin{array}{c}\mathrm{LC}_{50} \text { value } \\
\left(\mu \mathrm{g} \mathrm{cm}^{-2}\right)\end{array}$} & \multicolumn{2}{|c|}{ 95\% Confidence limits } & \multirow{2}{*}{$\chi^{2}$ Value (df) } & \multicolumn{2}{|c|}{ F-values } \\
\hline & & & Lower limit & Upper limit & & Duration & Doses \\
\hline \multirow{4}{*}{ Pet.E. } & 12 & 1.280 & 0.788 & 2.077 & $58.52(4)$ & \multirow{4}{*}{$0.814 \mathrm{~ns}$} & \multirow{4}{*}{$0.449 \mathrm{~ns}$} \\
\hline & 24 & 1.220 & 0.691 & 2.153 & $92.39(4)$ & & \\
\hline & 36 & 1.096 & 0.799 & 1.502 & $35.83(4)$ & & \\
\hline & 48 & 1.030 & 0.779 & 1.364 & $28.80(4)$ & & \\
\hline \multirow{4}{*}{$\mathrm{CHCl}_{3}$} & 12 & 3.249 & 2.839 & 3.717 & $1.41(3)$ & \multirow{4}{*}{$0.825 \mathrm{~ns}$} & \multirow{4}{*}{$45.102 * *$} \\
\hline & 24 & 2.990 & 2.663 & 3.358 & $0.75(3)$ & & \\
\hline & 36 & 2.526 & 2.313 & 2.759 & $3.28(4)$ & & \\
\hline & 48 & 2.204 & 2.000 & 2.429 & $5.28(4)$ & & \\
\hline \multirow{4}{*}{$\mathrm{MeOH}$} & 12 & 2.925 & 2.590 & 3.305 & $0.27(2)$ & \multirow{4}{*}{$0.738 \mathrm{~ns}$} & \multirow{4}{*}{$36.402 * *$} \\
\hline & 24 & 1.899 & 1.645 & 2.191 & $12.19(3)$ & & \\
\hline & 36 & 1.875 & 1.772 & 1.984 & $2.92(1)$ & & \\
\hline & 48 & 1.789 & 1.706 & 1.876 & - & & \\
\hline
\end{tabular}

All F-values are at 4df; $\mathrm{ns}=$ not significant; $* *=\mathrm{P}<0.01$.

Repellent effects: The repellency results are presented in Table 1 and 2. For E. nummularius $\left(\mathrm{CHCl}_{3}\right.$ and $\left.\mathrm{MeOH}\right)$ extracts mild activity $(\mathrm{P}<0.01)$ was established, while the Pet.E. extract did not show repellency at all (Table 1).

Table 2 Percent repulsion (PR) values and the arcsin transformed data of the Pet.E., $\mathrm{CHCl}_{3}$ and $\mathrm{MeOH}$ extracts of $E$. nummularius (whole plant) against $T$. castaneum adults.

\begin{tabular}{|c|c|c|c|c|c|c|}
\hline \multirow{3}{*}{$\begin{array}{l}\text { Type of } \\
\text { extracts }\end{array}$} & \multirow{3}{*}{$\begin{array}{c}\text { Dose } \\
\mathrm{mg} \mathrm{cm}\end{array}$} & \multicolumn{5}{|c|}{ Observation at regular intervals in hrs } \\
\hline & & 1 & 2 & 3 & 4 & 5 \\
\hline & & \multicolumn{5}{|c|}{$\begin{array}{c}\text { Percent repulsion } \mathrm{PR}=(\mathrm{Nc}-5) \times 20 \\
(\text { Arcsin transformed values for ANOVA) }\end{array}$} \\
\hline \multirow{5}{*}{ Pet.E } & 10 & $\begin{array}{l}100 \\
(90)\end{array}$ & $\begin{array}{l}100 \\
(90)\end{array}$ & $\begin{array}{l}100 \\
(90)\end{array}$ & $\begin{array}{c}80 \\
(63.43)\end{array}$ & $\begin{array}{l}100 \\
(90)\end{array}$ \\
\hline & 5 & $\begin{array}{l}100 \\
(90)\end{array}$ & $\begin{array}{l}100 \\
(90)\end{array}$ & $93.4(75.11)$ & $\begin{array}{l}100 \\
(90)\end{array}$ & $\begin{array}{l}100 \\
(90)\end{array}$ \\
\hline & 2.5 & $93.4(75.11)$ & $\begin{array}{l}100 \\
(90)\end{array}$ & $93.4(75.11)$ & $\begin{array}{l}100 \\
(90) \\
\end{array}$ & $\begin{array}{l}100 \\
(90) \\
\end{array}$ \\
\hline & 1.25 & $\begin{array}{c}93.4 \\
(75.11)\end{array}$ & $\begin{array}{l}100 \\
(90)\end{array}$ & $\begin{array}{l}100 \\
(90)\end{array}$ & $\begin{array}{l}100 \\
(90)\end{array}$ & $\begin{array}{l}100 \\
(90)\end{array}$ \\
\hline & 0.625 & $\begin{array}{l}100 \\
(90) \\
\end{array}$ & $\begin{array}{l}100 \\
(90) \\
\end{array}$ & $\begin{array}{l}100 \\
(90) \\
\end{array}$ & $\begin{array}{l}100 \\
(90) \\
\end{array}$ & $\begin{array}{l}100 \\
(90) \\
\end{array}$ \\
\hline \multirow{5}{*}{$\mathrm{CHCl}_{3}$} & 10 & $\begin{array}{l}100 \\
(90)\end{array}$ & $\begin{array}{l}100 \\
(90)\end{array}$ & $\begin{array}{l}100 \\
(90) \\
\end{array}$ & $\begin{array}{l}100 \\
(90)\end{array}$ & $\begin{array}{l}100 \\
(90)\end{array}$ \\
\hline & 5 & $53.4(46.95)$ & $66.6(54.70)$ & $\begin{array}{c}60 \\
(50.77) \\
\end{array}$ & $66.6(54.70)$ & $46.6(53.05)$ \\
\hline & 2.5 & $\begin{array}{c}93.4 \\
(75.11)\end{array}$ & $93.4(75.11)$ & $73.4(58.95)$ & $93.5(75.11)$ & $\begin{array}{c}80 \\
(63.43)\end{array}$ \\
\hline & 1.25 & $\begin{array}{l}100 \\
(90)\end{array}$ & $\begin{array}{l}100 \\
(90)\end{array}$ & $\begin{array}{c}100 \\
(90)\end{array}$ & $\begin{array}{c}100 \\
(90)\end{array}$ & $\begin{array}{l}100 \\
(90)\end{array}$ \\
\hline & 0.625 & $\begin{array}{c}60 \\
(50.77)\end{array}$ & $\begin{array}{c}66.6 \\
(54.70)\end{array}$ & $\begin{array}{l}100 \\
(90)\end{array}$ & $46.6(43.05)$ & $33.4(35.30)$ \\
\hline \multirow{5}{*}{$\mathrm{MeOH}$} & 10 & $\begin{array}{c}93.4 \\
(75.11)\end{array}$ & $\begin{array}{c}93.4 \\
(75.11)\end{array}$ & $\begin{array}{c}93.4 \\
(75.11)\end{array}$ & $\begin{array}{c}93.4 \\
(75.11)\end{array}$ & $\begin{array}{c}93.4 \\
(75.11)\end{array}$ \\
\hline & 5 & $\begin{array}{c}60 \\
(50.77) \\
\end{array}$ & $\begin{array}{c}46.6 \\
(43.05) \\
\end{array}$ & $\begin{array}{c}46.6 \\
(43.05) \\
\end{array}$ & $\begin{array}{c}46.6 \\
(43.05) \\
\end{array}$ & $\begin{array}{c}46.6 \\
(43.05) \\
\end{array}$ \\
\hline & 2.5 & $\begin{array}{l}100 \\
(90)\end{array}$ & $\begin{array}{l}100 \\
(90)\end{array}$ & $\begin{array}{l}100 \\
(90)\end{array}$ & $\begin{array}{l}100 \\
(90)\end{array}$ & $\begin{array}{l}100 \\
(90)\end{array}$ \\
\hline & 1.25 & $\begin{array}{l}100 \\
(90)\end{array}$ & $\begin{array}{l}100 \\
(90)\end{array}$ & $\begin{array}{l}100 \\
(90)\end{array}$ & $\begin{array}{l}100 \\
(90)\end{array}$ & $\begin{array}{l}100 \\
(90)\end{array}$ \\
\hline & 0.625 & $\begin{array}{c}60 \\
(50.77)\end{array}$ & $\begin{array}{l}100 \\
(90)\end{array}$ & $\begin{array}{c}73.4 \\
(58.95)\end{array}$ & $\begin{array}{c}80 \\
(58.95)\end{array}$ & $\begin{array}{c}73.4 \\
(58.95)\end{array}$ \\
\hline
\end{tabular}

Note: Higher percent repulsion for lower doses in comparison to corresponding higher doses was observed even in repetition of the same experiments, however the reason is yet to be investigated.

Lethal effect: The results of the brine shrimp lethality test for Pet.E., $\mathrm{CHCl}_{3}$ and $\mathrm{MeOH}$ extracts of $E$. nummularius (whole plant) are represented in Table 3. For Pet.E. extract the $\mathrm{LC}_{50}$ values ranged between 141.553 to $8.560 \mathrm{ppm}$; for the $\mathrm{CHCl}_{3}$ extract it was 
between 699.048 to $40.114 \mathrm{ppm}$, and for the $\mathrm{MeOH}$ extract the $\mathrm{LC}_{50}$ values ranged between 2336.982 to $137.0589 \mathrm{ppm}$. The intensity of activity could be arranged in a descending order: Pet.E. extract $>\mathrm{CHCl}_{3}$ extract> $\mathrm{MeOH}$ extract.

Table $3 \mathrm{LC}_{50}$ values (in ppm) of Pet.E., $\mathrm{CHCl}_{3}$ and $\mathrm{MeOH}$ extracts of $E$. nummularius against $A$. salina nauplii.

\begin{tabular}{ccccc}
\hline \multirow{2}{*}{$\begin{array}{c}\text { Type of } \\
\text { extract }\end{array}$} & \multicolumn{4}{c}{ Duration of exposure (in hrs) } \\
\cline { 2 - 5 } & $\mathbf{1 2}$ & $\mathbf{1 8}$ & $\mathbf{2 4}$ & $\mathbf{3 0}$ \\
\hline $\mathrm{Pet.E}$ & 141.553 & 98.941 & 24.684 & 8.560 \\
\hline $\mathrm{CHCl}_{3}$ & 699.048 & 366.663 & 326.0039 & 40.114 \\
\hline $\mathrm{MeOH}$ & 2336.982 & 1258.605 & 355.962 & 137.0589 \\
\hline
\end{tabular}

The record for biological activity of $E$. nummularius is very scanty. However, these findings receive supports from Pavithra et al., (2009), who studied antibacterial properties of E. nummularius. They found the susceptible organisms to the $\mathrm{MeOH}$ extract were Escherichia coli ( $\mathrm{MIC}=12.50 \mathrm{mg} / \mathrm{ml}$ ) and Bacillus subtilus $(\mathrm{MIC}=3.125 \mathrm{mg} / \mathrm{ml}$ ) and the most resistant strains were Staphylococcus aureus, Klebsiella pneumoniae and Pseudomonas aeruginosa. The same extracts exhibited radical scavenging activity with $\mathrm{IC}=50$ of $350 \mathrm{mg} / \mathrm{ml}$. Dash et al., (2003) evaluated the aqueous and hydroalcoholic extracts of E. nummularius that showed anthelmintic activity against adults of the Indian earthworm Pheretima posthuma. Ethnomedicinal uses of Evolvulus species have also been reported by many authors (poor sedative and anticonvulsant properties were reported by Chitralekha et al., 1964; reports for hysteria, to cure burns, cuts, wounds and scorpion stings have been made by Jain, 1991; activity to treat scabies mentioned by Manandhar and Manandhar, 2002; and the wound healing activity was reported by Saini et al., 2007). Medicinal use of $E$. nummularius indicates the potentially of this plant to have activity against certain causal agents or pathogens (microorganisms) and even functions on the components of human body for the cure of certain diseases.

Conclusion: The results from the study show that petroleum ether, chloroform and methanol extracts of E. nummularius have insecticidal activity, insect repellency and brine shrimp lethality; while from the previous researchers antibacterial and antioxidant activities have been reported which can be corroborated to the usage of this plant in traditional medicine. And thus, a comprehensive phytochemical analysis of the plant for its insecticidal and repellent components, as well as the physiological studies of the active ingredients are very much to be solicited for their effective use in the future pest control and pharmaceutical endeavours.
Acknoweledgements : The authors are grateful to the University Grants Commission (UGC) of Bangladesh and University of Rajshahi (R.U.), Bangladesh for providing research grants. The Chairman of the Department of Zoology, R.U. deserves special thanks for providing laboratory facilities.

\section{References}

Abbott WS. 1925. A method of computing the effectiveness of an insecticide, J. Econ. Ent. 18: 265-267.

Anon. 1948. The Wealth of India. CSIR, New Delhi, India. 1: 62-63.

Anon.1959. The Wealth of India. CSIR, New Delhi, India. 5: 314-315.

Borchers AT, Hackman RM, Keen CL, Stern JS, Gershwin ME. 1997. Complementary medicine: A review of immunomodulatory effects of Chinese herbal medicines. American J. Clinic. Nutri. 66: 1303-1312.

Busvine JR. 1971. A critical review of the techniques for testing insecticides. Commonwealth Agricultural Bureau, London, $345 \mathrm{pp}$.

Chitralekha C, Dey PK, Dey CD. 1964. Pharmacological screening of Valeriana wallichi, Lallemantia royleana, Breynia rhamnoides and Evolvulus nummularius for sedative and anticonvulsant principle, Nature Wissenschaften 51: 411

Dash GK, Bijayini M, Panda A, Patro CP, Ganapathy, S. 2003. Antihelmintic activity of Evolvulus nummularius. Indian J. Nat. Prod. 19: 24-26.

Dubey NK, Rajesh K, Pramila T. 2004. Global promotion of herbal medicine: India's opportunity. Curr. Sci. 86: 37-41.

Finney DJ. 1947. Probit analysis: a statistical treatment of the sigmoid response curve. Cambridge University Press, London. 333 pp.

Jain SK. 1991. Dictionary of Indian folk medicine and ethnobotany, New Deep Publication, 92pp.

Manandhar NP, Manandhar, S. 2002. Plants and People of Nepal. Timber Press Inc., Portland, USA, p. 230.

McDonald LL, Guy RH, Speirs RD. 1970. Preliminary evaluation of new candidate materials as toxicants, repellents and attractants against stored-product insects. Marketing Research Report No. 882. Agricultural Research Service, US Department of Agriculture, Washington, DC.

Pavithra PS, Srevidya N, Verma RS. 2009. Antibacterial and antioxidant activity of Methanol extract of Evolvulus nummularius. Indian J. Pharmacol. 41: 233-336.

Saini V, Kinger HK, Sharma DK, Ahuja N, Middha A, Gupta VB. 2007. Wound healing activity of Evolvulus numularius Linn. Asian J. Chem.19: 5772-4.

Talukder FA, Howse PE. 1993. Deterrent and insecticidal effects of extracts of pithraj, Aphanamixis polystachya (Meliaceae) against Tribolium castaueum in storage. J. Chem. Ecol. 19: 2463-2471.

Talukder FA, Howse PE. 1994. Repellent, toxic and food protectant effect of Pithraj, Aphanamixis polystachya extracts against pulse beetle, Callosobruchus chinensis in storage. J. Chem. Ecol. 20: 899-908.

Talukder FA, Howse PE. 1995. Evaluation of Aphanamixis polystachya as a source of repellents, antifeedants, toxicants and protectants in storage against Tribolium castaneum (Herbs). J. Stored. Prod. Res. 31: 55-61.

Manuscript receive on 1 December 2013 and revised on 21 December 2013 